\title{
Xylanilyticolides A-C, Three New Compounds from Cultures of the Actinomycete Promicromonospora xylanilytica YIM 61515
}

\author{
Zhen-Xiong Wang ${ }^{1} \cdot$ Shen $\mathrm{Qin}^{2} \cdot \mathrm{Li}^{-H u a} \mathrm{Xu}^{2} \cdot$ He-Ping Chen ${ }^{1} \cdot$ Huan Sun ${ }^{1} \cdot$ \\ Rong Huang ${ }^{1} \cdot{\text { Zheng-Hui } \mathrm{Li}^{1} \cdot \text { Tao Feng }^{1} \text { (I) Ji-Kai Liu }}^{1}$
}

Received: 27 December 2017 / Accepted: 19 January 2018/Published online: 13 February 2018

(C) The Author(s) 2018. This article is an open access publication

\section{Abstract}

Three new lactones, xylanilyticolides A-C (1-3), were isolated from cultures of the actinomycete Promicromonospora xylanilytica YIM 61515. Their structures were elucidated by 1D and 2D NMR spectroscopic data in conjunction with HRESIMS analysis. Compound 1 exhibited potent cytotoxicities against five human cancer cell lines HL-60, A-549, SMMC-7721, MCF-7 and SW480 with the $\mathrm{IC}_{50}$ values of $3.9,15.2,11.2,5.9$, and $4.7 \mu \mathrm{M}$, respectively.

\section{Graphical Abstract}
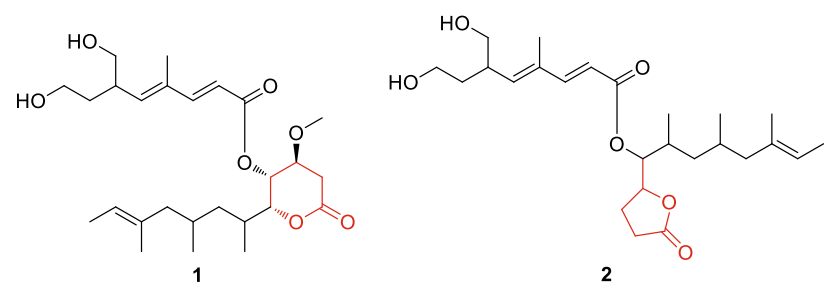

Keywords Actinomycete $\cdot$ Promicromonospora xylanilytica $\cdot$ Xylanilyticolides $\cdot$ Cytotoxicity

\section{Introduction}

Actinomycetes are well-known for their ability to produce natural products with structural complexity and diverse biological activities [1, 2]. Salinomycin, isolated from the culture broth of an actinomycete strain of Streptomyces,

Electronic supplementary material The online version of this article (https://doi.org/10.1007/s13659-018-0154-1) contains supplementary material, which is available to authorized users.

Tao Feng

tfeng@mail.scuec.edu.cn

$\triangle$ Ji-Kai Liu

jkliu@mail.kib.ac.cn

1 School of Pharmaceutical Sciences, South-Central University for Nationalities, Wuhan 430074, China

2 Yunnan Institute of Microbiology, Yunnan University, Kunming 650091, China shows remarkable toxicity for cancer stem cells [3, 4]. Actinomycetes, including some rare genera of actinomycetes [5], are also valuable sources in the discovery of natural antibiotics [6].

Since the genus Promicromonospora was proposed by Krasil'nikov et al. in 1961 [7], there has been only one report of chemical investigation on this genus with discovery of a novel macrocyclic dilactone from Promicromonospora sp. RL26 in 2011 [8]. Herein, the investigation of secondary metabolites from the Promicromonospora xylanilytica YIM 61515 led to the discovery of three new products, xylanilyticolides A-C (1-3) (Fig. 1) [9]. Their structures were elucidated by analyses of 1D and 2D NMR spectroscopic methods. Their cytotoxicities against five human cancer cell lines were evaluated. Report herein are the isolation, structural elucidation and cytotoxicities of them. 


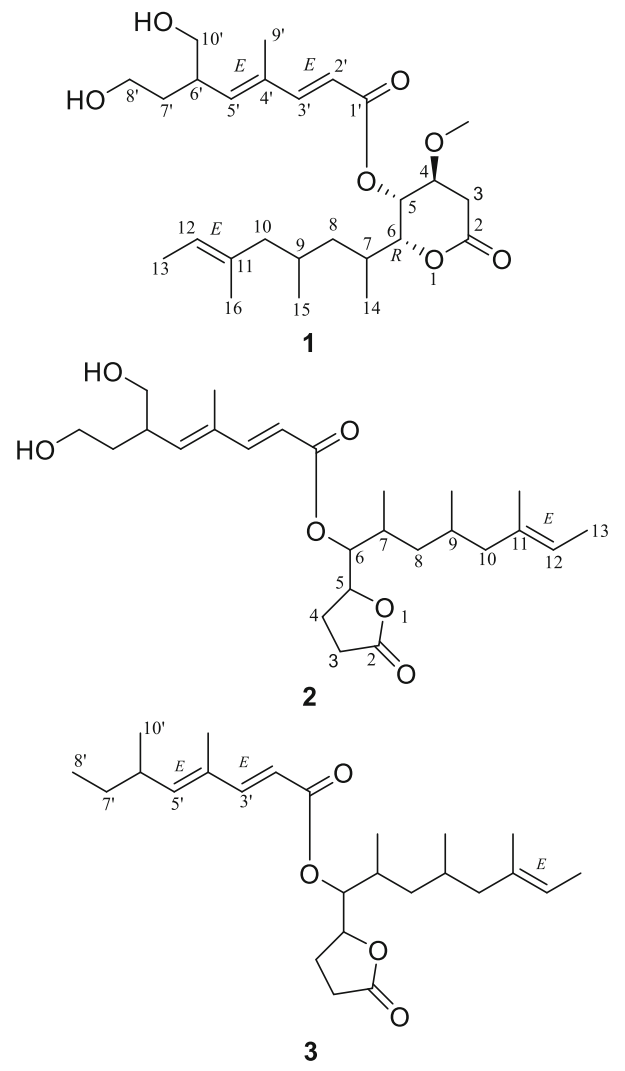

Fig. 1 Structures of compounds $\mathbf{1}-\mathbf{3}$

\section{Results and Discussion}

\subsection{Structural Elucidation of Xylanilyticolides A-C (1-3)}

Xylanilyticolide A (1), obtained as colorless oil, had a formula of $\mathrm{C}_{26} \mathrm{H}_{42} \mathrm{O}_{7}$ deduced by HRESIMS sodium adduct ion peak at $\mathrm{m} / \mathrm{z} 489.2822[\mathrm{M}+\mathrm{Na}]^{+}$(calcd. for $\mathrm{C}_{26} \mathrm{H}_{42} \mathrm{O}_{7} \mathrm{Na}$, 489.2823), requiring six degrees of unsaturation. The ${ }^{1} \mathrm{H}$ NMR data displayed three methyl singlets at $\delta_{\mathrm{H}} 1.84\left(\mathrm{H}-9^{\prime}\right), 1.52(\mathrm{H}-16), 3.44(\mathrm{OMe})$, three methyl doublets at $\delta_{\mathrm{H}} 1.54(\mathrm{H}-13, \mathrm{~d}, J=6.2 \mathrm{~Hz}), 1.09(\mathrm{H}-14, \mathrm{~d}, J=$ $6.6 \mathrm{~Hz}), 0.77(\mathrm{H}-15, \mathrm{~d}, J=6.6 \mathrm{~Hz})$, and two olefinic protons at $\delta_{\mathrm{H}} 5.81\left(\mathrm{H}-2^{\prime}, \mathrm{d}, J=15.7 \mathrm{~Hz}\right), 7.34\left(\mathrm{H}-3^{\prime}, \mathrm{d}, J=\right.$ $15.7 \mathrm{~Hz}$ ). The ${ }^{13} \mathrm{C}$ NMR and DEPT spectra (Table 1) exhibited signals for 26 carbon atoms, including two carbonyls $\left(\delta_{\mathrm{C}} 165.9,169.5\right)$, six olefinic carbons $\left(\delta_{\mathrm{C}} 115.3\right.$, $120.2,134.4,134.8,143.7,151.1)$, six methyls $\left(\delta_{\mathrm{C}} 12.8\right.$, $13.5,15.7,16.1,20.7,57.3)$. The above data coupled with a literature survey indicated that compound $\mathbf{1}$ was analogous to the reported compound rasfonin, an apoptosis inducer in Ras-dependent cells isolated from the fungus Talaromyces sp. [10]. Comparing with the reported structure, the two distinct differences in compound $\mathbf{1}$ were the reduction of olefinic bond in C-3 and the substitution of a methoxy in
C-4. The ${ }^{1} \mathrm{H}-{ }^{1} \mathrm{H}$ COSY correlations between $\mathrm{H}-3\left(\delta_{\mathrm{H}} 2.79\right.$, $2.67) / \mathrm{H}-4\left(\delta_{\mathrm{H}} 3.70\right), \mathrm{H}-4 / \mathrm{H}-5\left(\delta_{\mathrm{H}} 5.29\right)$, and HMBC correlation from $-\mathrm{OCH}_{3}\left(\delta_{\mathrm{H}} 3.44\right)$ to $\mathrm{C}-4\left(\delta_{\mathrm{C}} 74.1\right)$, from $\mathrm{H}-3$ $\left(\delta_{\mathrm{H}} 2.79,2.67\right)$ and $\mathrm{H}-4$ to $\mathrm{C}-2\left(\delta_{\mathrm{C}} 169.5\right)$ supported these changes (Fig. 2).

$\Delta^{2^{\prime}}$ was established as $E$ configuration, which could be confirmed by the coupling constant (see Table 1). Verified by the ROESY correlation between $\mathrm{H}-3^{\prime}\left(\delta_{\mathrm{H}} 7.34\right) / \mathrm{H}-5^{\prime}\left(\delta_{\mathrm{H}}\right.$ 5.78), $\Delta^{4^{\prime}}$ could identified as $E$ configuration. $\Delta^{11}$ was $E$ configuration, which could be identified by the $\mathrm{H}-12\left(\delta_{\mathrm{H}}\right.$ $5.12) / \mathrm{H}-10\left(\delta_{\mathrm{H}} 2.00,1.44\right)$ ROESY correlation. From the Newman projection (Fig. 3), the $J_{\mathrm{H}-3 / \mathrm{H}-4}$ of $3.2 \mathrm{~Hz}$ and $5.5 \mathrm{~Hz}, J_{\mathrm{H}-4 / \mathrm{H}-5}$ of $3.2 \mathrm{~Hz}, J_{\mathrm{H}-6 / \mathrm{H}-5}$ of $1.5 \mathrm{~Hz}$ as well as H-5/H-6 ROESY correlation were observed, which could confirmed the relative configuration of the lactone ring as $4 S^{*}, 5 R^{*}, 6 R^{*}$. However, the stereoconfigurations of $\mathrm{C}-7$, C-9, and C- 6 ' can't be determined by the ROESY experiment. The calculated ECD and single crystal X-ray diffraction were also in failure. Therefore, the structure of compound 1 was deduced as xylanilyticolide A.

Compound 2 was obtained as colorless oil. Its molecular formula was assigned as $\mathrm{C}_{25} \mathrm{H}_{40} \mathrm{O}_{6}$ by HRESIMS ion peak at $\mathrm{m} / z \quad 459.2723[\mathrm{M}+\mathrm{Na}]^{+}$(calcd. for $\mathrm{C}_{25} \mathrm{H}_{40} \mathrm{O}_{6} \mathrm{Na}$, 459.2717), implying the presence of six degrees of unsaturation. The ${ }^{1} \mathrm{H}$ NMR spectrum of 2 illustrated the presence of two methyl singlets at $\delta_{\mathrm{H}} 1.54(\mathrm{H}-16)$ and $1.87(\mathrm{H}-$ $\left.9^{\prime}\right)$; two oxygenated methylenes at $\delta_{\mathrm{H}} 3.59 / 3.49\left(\mathrm{H}-8^{\prime}\right)$, $3.53\left(\mathrm{H}-10^{\prime}\right)$; four olefinic protons at $\delta_{\mathrm{H}} 5.87\left(\mathrm{H}-2^{\prime}\right), 7.34$ $\left(\mathrm{H}-3^{\prime}\right), 5.84\left(\mathrm{H}-5^{\prime}\right), 5.15(\mathrm{H}-12)$; and two oxygenated methines at $\delta_{\mathrm{H}} 4.79(\mathrm{H}-5), 5.06(\mathrm{H}-6)$. Combined ${ }^{13} \mathrm{C} \mathrm{NMR}$ data (Table 1) and HSQC spectrum indicated the presence of five methyls, seven methylenes, five saturated methines, four olefinic methines, two fully substituted carbons $\left(\delta_{\mathrm{C}}\right.$ 135.2 and 134.7), and two ketone carbons $\left(\delta_{\mathrm{C}} 176.8\right.$ and 167.0). The ${ }^{1} \mathrm{H}$ and ${ }^{13} \mathrm{C}$ chemical shifts of compound 2 (Table 1) were highly similar to those of $\mathbf{1}$, except the $\delta$ lactone moiety in $\mathbf{1}$ was replaced by a $\gamma$-lactone moiety in 2, which could be verified by the HMBC correlation between $\mathrm{H}-5\left(\delta_{\mathrm{H}} 4.79\right) / \mathrm{C}-2\left(\delta_{\mathrm{C}} 176.8\right)$ and ${ }^{1} \mathrm{H}-{ }^{1} \mathrm{H}$ COSY correlations of H-6 $\left(\delta_{\mathrm{H}} 5.06\right) / \mathrm{H}-5\left(\delta_{\mathrm{H}} 4.79\right), \mathrm{H}-5\left(\delta_{\mathrm{H}} 4.79\right) /$ $\mathrm{H}-4\left(\delta_{\mathrm{H}} 2.38,1.98\right), \mathrm{H}-4\left(\delta_{\mathrm{H}} 2.38,1.98\right) / \mathrm{H}-3\left(\delta_{\mathrm{H}} 2.53,2.47\right)$ of compound 2 (Fig. 2). The $E / Z$ configurations of the double bonds in compound $\mathbf{2}$ were deduced as the same with those of compound $\mathbf{1}$ by ROESY correlations of $\mathrm{H}-9^{\prime}$ with $\mathrm{H}-6^{\prime}$ and $\mathrm{H}-10^{\prime}$. However, the stereochemistry of C-7, C-9, and C-6' can't be established currently. The structure of compound $\mathbf{2}$ was, therefore, identified as xylanilyticolide B.

The molecular of compound $\mathbf{3}$ was established by HRESIMS ion peak at $\mathrm{m} / \mathrm{z} 427.2817[\mathrm{M}+\mathrm{Na}]^{+}$(calcd. for $\mathrm{C}_{25} \mathrm{H}_{40} \mathrm{O}_{4} \mathrm{Na}$, 427.2819). Detailed analyses of ${ }^{1} \mathrm{H}$ and ${ }^{13} \mathrm{C}$ NMR data of compound $\mathbf{3}$ revealed that compound $\mathbf{3}$ had the same skeleton with that of compound 2 . The main 
Table $1{ }^{1} \mathrm{H}(600 \mathrm{MHz})$ and ${ }^{13} \mathrm{C}(150 \mathrm{MHz})$ NMR spectroscopic data of $\mathbf{1}-\mathbf{3}(\delta$ in ppm, $J$ in $\mathrm{Hz})$

\begin{tabular}{|c|c|c|c|c|c|c|}
\hline \multirow[t]{2}{*}{ No. } & \multicolumn{2}{|l|}{$\mathbf{1}\left(\mathrm{CDCl}_{3}\right)$} & \multicolumn{2}{|c|}{2 (acetone- $d_{6}$ ) } & \multicolumn{2}{|c|}{3 (methanol- $\left.d_{4}\right)$} \\
\hline & $\delta_{\mathrm{C}}$, type & $\delta_{\mathrm{H}}$ & $\delta_{\mathrm{C}}$, type & $\delta_{\mathrm{H}}$ & $\delta_{\mathrm{C}}$, type & $\delta_{\mathrm{H}}$ \\
\hline 2 & $169.5, \mathrm{C}$ & & $176.8, \mathrm{C}$ & & $179.5, \mathrm{C}$ & \\
\hline 3 & $32.8, \mathrm{CH}_{2}$ & $\begin{array}{l}2.79(17.8,5.5) \\
2.67(17.8,3.2)\end{array}$ & $28.8, \mathrm{CH}_{2}$ & $\begin{array}{l}2.53 \text {, ddd }(17.5,9.5,9.5) \\
2.47 \text {, ddd }(17.5,9.5,4.5)\end{array}$ & $29.3, \mathrm{CH}_{2}$ & $\begin{array}{l}2.58 \text {, ddd }(17.5,10.0,8.9) \\
2.49 \text {, overlapped }\end{array}$ \\
\hline 4 & 74.1, CH & 3.70 , ddd $(5.5,3.2,3.2)$ & $25.2, \mathrm{CH}_{2}$ & $2.38, \mathrm{~m} ; 1.98, \mathrm{~m}$ & $25.5, \mathrm{CH}_{2}$ & $2.35, \mathrm{~m} ; 1.95, \mathrm{~m}$ \\
\hline 5 & $66.1, \mathrm{CH}$ & 5.29, dd $(3.2,1.5)$ & $80.5, \mathrm{CH}$ & 4.79 , ddd $(7.4,7.4,6.5)$ & $81.9, \mathrm{CH}$ & 4.81 , ddd $(7.4,7.4,6.4)$ \\
\hline 6 & $81.3, \mathrm{CH}$ & $4.31, \mathrm{dd}(8.7,1.5)$ & 76.7, $\mathrm{CH}$ & $5.06, \mathrm{dd}(6.5,4.0)$ & 77.3, $\mathrm{CH}$ & $5.04, \mathrm{dd}(6.4,4.0)$ \\
\hline 7 & $31.7, \mathrm{CH}$ & $1.99, \mathrm{~m}$ & $31.9, \mathrm{CH}$ & $2.03, \mathrm{~m}$ & $32.4, \mathrm{CH}$ & 2.01, overlapped \\
\hline 8 & $40, \mathrm{CH}_{2}$ & $\begin{array}{l}1.22, \text { br. dd }(13.3,5.0) \\
1.01, \text { ddd }(13.3,9.0,3.8)\end{array}$ & $41.7, \mathrm{CH}_{2}$ & $\begin{array}{l}\text { 1.36, ddd }(14.4,7.6,6.3) \\
0.94, \mathrm{~m}\end{array}$ & $41.9, \mathrm{CH}_{2}$ & $\begin{array}{l}1.37, \mathrm{~m} \\
0.91, \mathrm{~m}\end{array}$ \\
\hline 9 & $27.9, \mathrm{CH}$ & $1.67, \mathrm{~m}$ & $28.3, \mathrm{CH}$ & $1.79, \mathrm{~m}$ & $28.7, \mathrm{CH}$ & $1.75, \mathrm{~m}$ \\
\hline 10 & $46.4, \mathrm{CH}_{2}$ & $\begin{array}{l}2.00, \text { overlapped } \\
1.44, \text { dd }(13.1,9.8)\end{array}$ & $48.5, \mathrm{CH}_{2}$ & $\begin{array}{l}\text { 1.97, overlapped } \\
1.68, \text { dd }(13.0,8.5)\end{array}$ & 29.6, $\mathrm{CH}_{2}$ & $\begin{array}{l}\text { 1.92, overlapped } \\
1.72, \mathrm{~m}\end{array}$ \\
\hline 11 & $134.4, \mathrm{C}$ & & $135.2, \mathrm{C}$ & & $135.5, \mathrm{C}$ & \\
\hline 12 & $120.2, \mathrm{CH}$ & $5.12, \mathrm{q}(6.2)$ & $120.5, \mathrm{CH}$ & $5.15, \mathrm{q}(6.6)$ & $121.0, \mathrm{CH}$ & $5.15, \mathrm{q}(6.6)$ \\
\hline 13 & $13.5, \mathrm{CH}_{3}$ & $1.54, \mathrm{~d}(6.2)$ & $13.4, \mathrm{CH}_{3}$ & $1.52, \mathrm{~d}(6.6)$ & $13.5, \mathrm{CH}_{3}$ & $1.53, \mathrm{~d}(6.6)$ \\
\hline 14 & $16.1, \mathrm{CH}_{3}$ & $1.09, \mathrm{~d}(6.6)$ & 15.6, $\mathrm{CH}_{3}$ & $0.98, \mathrm{~d}(6.8)$ & $15.7, \mathrm{CH}_{3}$ & $0.99, \mathrm{~d}(6.8)$ \\
\hline 15 & $20.7, \mathrm{CH}_{3}$ & $0.77, \mathrm{~d}(6.6)$ & $20.2, \mathrm{CH}_{3}$ & $0.82, \mathrm{~d}(6.5)$ & $20.4, \mathrm{CH}_{3}$ & $0.84, \mathrm{~d}(6.3)$ \\
\hline 16 & $15.7, \mathrm{CH}_{3}$ & $1.52, \mathrm{~s}$ & $15.6, \mathrm{CH}_{3}$ & $1.54, \mathrm{~s}$ & $15.7, \mathrm{CH}_{3}$ & $1.54, \mathrm{~s}$ \\
\hline $1^{\prime}$ & 165.9, C & & 167.0, C & & $168.5, \mathrm{C}$ & \\
\hline $2^{\prime}$ & $115.3, \mathrm{CH}$ & $5.81, \mathrm{~d}(15.7)$ & $116.2, \mathrm{CH}$ & $5.87, \mathrm{~d}(15.7)$ & $115.9, \mathrm{CH}$ & $5.85, \mathrm{~d}(15.6)$ \\
\hline $3^{\prime}$ & 151.1, CH & $7.34, \mathrm{~d}(15.7)$ & $150.9, \mathrm{CH}$ & $7.34, \mathrm{~d}(15.7)$ & $150.3, \mathrm{CH}$ & $7.34, \mathrm{~d}(15.6)$ \\
\hline $4^{\prime}$ & $134.8, \mathrm{C}$ & & 134.7, C & & 133.1, C & \\
\hline $5^{\prime}$ & 143.7, CH & $5.78, \mathrm{~d}(10.0)$ & 145.6, CH & $5.84, \mathrm{~d}(10.0)$ & $152.2, \mathrm{CH}$ & $5.73, \mathrm{~d}(10.0)$ \\
\hline $6^{\prime}$ & $39.4, \mathrm{CH}$ & $2.89, \mathrm{~m}$ & $39.8, \mathrm{CH}$ & $2.89, \mathrm{~m}$ & $36.2, \mathrm{CH}$ & $2.52, \mathrm{~m}$ \\
\hline $7^{\prime}$ & $34.9, \mathrm{CH}_{2}$ & $1.80, \mathrm{~m} ; 1.61, \mathrm{~m}$ & $35.7, \mathrm{CH}_{2}$ & $1.82, \mathrm{~m} ; 1.48, \mathrm{~m}$ & $31.1, \mathrm{CH}_{2}$ & $1.46, \mathrm{~m} ; 1.33, \mathrm{~m}$ \\
\hline $8^{\prime}$ & $60.8, \mathrm{CH}_{2}$ & $3.74, \mathrm{~m} ; 3.61$, overlapped & $60.4, \mathrm{CH}_{2}$ & $3.59, \mathrm{~m} ; 3.49, \mathrm{~m}$ & $12.3, \mathrm{CH}_{3}$ & $0.87, \mathrm{t}(7.4)$ \\
\hline $9^{\prime}$ & $12.8, \mathrm{CH}_{3}$ & $1.84, \mathrm{~s}$ & $12.8, \mathrm{CH}_{3}$ & $1.87, \mathrm{~s}$ & $12.6, \mathrm{CH}_{3}$ & $1.83, \mathrm{~s}$ \\
\hline $10^{\prime}$ & $66.0, \mathrm{CH}_{2}$ & $\begin{array}{l}3.63 \text {, overlapped } \\
3.58, \text { overlapped }\end{array}$ & 66.0, $\mathrm{CH}_{2}$ & $3.53, \mathrm{~s}, 2 \mathrm{H}$ & $20.5, \mathrm{CH}_{3}$ & $1.02, \mathrm{~d}(6.6)$ \\
\hline$-\mathrm{OMe}$ & $57.3, \mathrm{CH}_{3}$ & $3.44, \mathrm{~s}$ & & & & \\
\hline
\end{tabular}

difference was that $\mathrm{C}-8^{\prime}$ and $\mathrm{C}-10^{\prime}$ in compound $\mathbf{3}$ was two methyl carbons rather than two hydroxymethyl groups in $\mathbf{2}$. Detailed analysis of 2D NMR data suggested that other parts of $\mathbf{3}$ were the same to those of $\mathbf{2}$. Hence the structure of $\mathbf{3}$ was elucidated as xylanilyticolide $\mathrm{C}$.

\subsection{Cytotoxicity Assays}

Compounds 1-3 were evaluated for their cytotoxicity activity utilizing an MTS cytotoxicity assay in vitro (DDP was used as a positive control). Compound $\mathbf{1}$ showed potent inhibitory activity against HL-60, A-549, SMMC$7721, \mathrm{MCF}-7$ and SW480 cells lines with the $\mathrm{IC}_{50}$ values of $3.9,15.2,11.2,5.9,4.7 \mu \mathrm{M}$, respectively (Table 2), while compounds $\mathbf{2}$ and $\mathbf{3}$ showed no significant cytotoxicity activity under the concentration of $40 \mu \mathrm{M}$.

\section{Experimental}

\subsection{General Experimental Procedures}

Optical rotations were recorded on a JASCO P-1020 digital polari-meter (Horiba, Kyoto, Japan). UV/Vis spectra were obtained using a Shimadzu UV2401PC spectrometer (Shimadzu, Kyoto, Japan). 1D and 2D NMR spectra were measured on a Bruker Avance III $600 \mathrm{MHz}$ spectrometers (Bruker Biospin GmbH, Karlsruhe, Germany). HRESIMS were recorded on an Agilent 6200 Q-TOF MS system (Agilent Technologies, Santa Clara, CA, USA). Silica gel (200-300 mesh, Qingdao Haiyang Chemical Co., Ltd., P. R. China) was used for chromatography column. Size exclusion chromatography was performed on Sephadex LH-20 (GE, Healthcare) columns. MPLC separation was 
<smiles>CC=C(C)CC(C)CC(C)C1OC(=O)CCC2(CCO2)C1OC(=O)C=CC(C)=CC(CO)CCO</smiles>

$\longrightarrow{ }^{1} \mathrm{H}-{ }^{1} \mathrm{H}$ COSY $\overparen{\mathrm{H}} \mathrm{C}$ HMBC

Fig. 2 Key 2D NMR correlations of compounds 1 and 2

conducted on a Biotage Isolera One using a Spherical C18 column. Semi-preparative HPLC was performed on an Agilent 1260 liquid chromatography system equipped with Zorbax SB-C18 columns $(9.4 \mathrm{~mm} \times 150 \mathrm{~mm}$, particle size $5 \mu \mathrm{m})$.

\subsection{Actinomycete Materials and Culture Condition}

The strain was identified as P. xylanilytica YIM 61515 by Prof. Shen Qin of Yunnan University. A voucher strain (HFC: 20160920-4A) was deposited at the School of Pharmaceutical Sciences, South-Central University for Nationalities, China.

The $P$. xylanilytica YIM 61515 strain was cultured in seed medium (glucose $20 \mathrm{~g}$, peptone $2 \mathrm{~g}$, yeast extract $2 \mathrm{~g}$, soluble starch $5 \mathrm{~g}, \mathrm{~K}_{2} \mathrm{HPO}_{4} 0.5 \mathrm{~g}, \mathrm{MgSO}_{4} 0.5 \mathrm{~g}, \mathrm{NaCl} 4 \mathrm{~g}$, $\mathrm{CaCO}_{3} 2 \mathrm{~g}$ in $1 \mathrm{~L}$ of deionized water, the $\mathrm{pH}$ was adjusted to 7.8 before autoclaving) at $25^{\circ} \mathrm{C}$ with shaking at $150 \mathrm{rpm}$ for 7 days. Then inoculated the seed culture into 50 Erlenmeyer flasks $(500 \mathrm{~mL})$, previously sterilized by autoclaving, each containing $100 \mathrm{~g}$ of rice and $100 \mathrm{~mL}$ of
Table 2 Cytotoxicities of $\mathbf{1}$ against five human cancer cell lines $\left(\mathrm{IC}_{50}\right.$ $\mu \mathrm{M})$

\begin{tabular}{llllcc}
\hline Compound & HL-60 & A-549 & SMMC-7721 & MCF-7 & SW480 \\
\hline $\mathbf{1}$ & 3.9 & 15.2 & 11.2 & 5.9 & 4.7 \\
DDP $^{\mathrm{a}}$ & 4.6 & 27.4 & 28.0 & 29.3 & 30.0 \\
\hline
\end{tabular}

${ }^{\text {a }}$ Positive control

distilled water. All flasks were incubated at $25{ }^{\circ} \mathrm{C}$ for 70 days.

\subsection{Extraction and Isolation}

Each flask added $200 \mathrm{~mL}$ of EtOAc, and the culture was homogenized. The suspension was extracted repeatedly with EtOAc $(4 \times 10 \mathrm{~L})$, and the organic layer was evaporated to dryness under vacuum to yield a crude extract (49 g), which was fractionated by MPLC with a stepwise gradient of $\mathrm{MeOH} / \mathrm{H}_{2} \mathrm{O}$ (v/v 10:90, 40:60, 70:30, 90:10, 100:0) to give nine fractions (Fr. A-J).

Fr. D (1.87 g) was subjected to 200-300 mesh silica gel column with stepwise elution petroleum ether-EtOAc (8:1-2:1) to obtain nine sub-fractions (D1-D9) based on TLC analyses. The third fraction Fr. D3 was purified by semi-preparative HPLC using a gradient elution (MeCN/ $\mathrm{H}_{2} \mathrm{O} 50: 502.5 \mathrm{~mL} / \mathrm{min}$ ) to give compound 1 (4.4 $\mathrm{mg}$, colorless oil).

Fr. E (508 mg) was separated on Sephadex LH-20 $(\mathrm{MeOH})$ to give four fractions (E1-E4), and compound 2 (3.9 mg) was obtained from Fr. E2 (66.9 mg) which purified with semi-preparative HPLC using an isocratic elution (MeCN/ $\mathrm{H}_{2} \mathrm{O}$ 55:45, $\left.3 \mathrm{~mL} / \mathrm{min}\right)$.

Fr. F (1.9 g) was subjected to 200-300 mesh silica gel column eluting with petroleum ether-EtOAc 4:1 to give 5 fractions (F1-F5). Fr. F3 (98.4 mg) was subjected to semiprep HPLC with isocratic elution $\left(\mathrm{MeCN} / \mathrm{H}_{2} \mathrm{O}\right.$ 90:10, $3 \mathrm{~mL} / \mathrm{min})$ to give compound $3(0.8 \mathrm{mg})$.

Xylanilyticolide $A(\mathbf{1})$ colorless oil, $[\alpha]_{\mathrm{D}}^{24} 8.2$ (c 0.11 , $\mathrm{MeOH})$; UV (MeOH) $\lambda$ max $(\log \varepsilon) 202$ (3.90), 269 (4.31) $\mathrm{nm} ;{ }^{1} \mathrm{H}$ and ${ }^{13} \mathrm{C}$ NMR data, see Table 1 . HRESIMS $\mathrm{m} / \mathrm{z}$

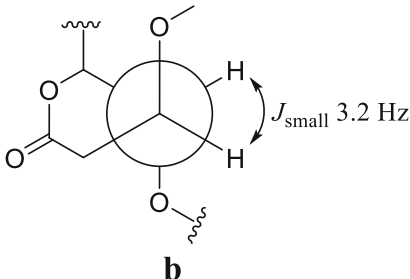

C4-C5 Newman projection

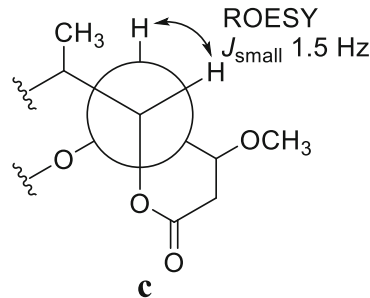

C6-C5 Newman projection 
489.2822 $[\mathrm{M}+\mathrm{Na}]^{+}$(calcd. for $\mathrm{C}_{26} \mathrm{H}_{42} \mathrm{O}_{7} \mathrm{Na}$, 489.2823).

Xylanilyticolide $B(\mathbf{2})$ colorless oil, $[\alpha]_{\mathrm{D}}^{24} 1.7$ ( c 0.13 , $\mathrm{MeOH}) ; \mathrm{UV}(\mathrm{MeOH}) \lambda_{\max }(\log \varepsilon) 202$ (3.85), 268 (4.31) $\mathrm{nm} ;{ }^{1} \mathrm{H}$ and ${ }^{13} \mathrm{C}$ NMR data, see Table 1. HRESIMS $m / z \quad 459.2723[\mathrm{M}+\mathrm{Na}]^{+}$(calcd. for $\mathrm{C}_{25} \mathrm{H}_{40} \mathrm{O}_{6} \mathrm{Na}$, 459.2717).

Xylanilyticolide $C(\mathbf{3})$ colorless oil, $[\alpha]_{\mathrm{D}}^{24} 4.5$ (c 0.07 , $\mathrm{MeOH}) ; \mathrm{UV}(\mathrm{MeOH}) \lambda_{\max }(\log \varepsilon) 202$ (3.15), 262 (3.77), 544 (1.35) nm; ${ }^{1} \mathrm{H}$ and ${ }^{13} \mathrm{C}$ NMR data, see Table 1. HRESIMS $m / z \quad 427.2817 \quad[\mathrm{M}+\mathrm{Na}]^{+}$(calcd. for $\mathrm{C}_{25} \mathrm{H}_{40} \mathrm{O}_{4} \mathrm{Na}$, 427.2819).

\subsection{Cytotoxicity}

Five human cancer cell lines, breast cancer SK-BR-3, hepatocellular carcinoma SMMC-7721, human myeloid leukemia HL-60, pancreatic cancer PANC-1, and lung cancer A-549 were used. Cells were cultured in RPMI1640 or in DMEM medium (Hyclone, Logan, UT, USA), supplemented with 10\% fetal bovine serum (Hyclone, USA) in $5 \% \mathrm{CO}_{2}$ at $37{ }^{\circ} \mathrm{C}$. The cytotoxicity assay was performed according to 3-(4,5-dimethylthiazol-2-yl)-2,5diphenyl tetrazolium bromide (MTT) method in 96-well microplates [11]. Briefly, $100 \mu \mathrm{L}$ of adherent cells were seeded into each well of 96-well cell culture plates and allowed to adhere for $12 \mathrm{~h}$ before addition of test compounds, while suspended cells were seeded just before drug addition with initial density of $1 \times 10^{5}$ cells $/ \mathrm{mL}$. Each tumor cell line was exposed to the test compound at concentrations of $0.0625,0.32,1.6,8$, and $40 \mu \mathrm{M}$ in triplicates for $48 \mathrm{~h}$, and all tests were done in twice with cisplatin (Sigma, Los Angeles, USA) as a positive control. After compound treatment, cell viability was detected and a cell growth curve was graphed. $\mathrm{IC}_{50}$ values were calculated by Reed and Muench's method [12].

Acknowledgements This work was financially supported by National Natural Science Foundation of China (81561148013, 21502239), the
Key Projects of Technological Innovation of Hubei Province (No. 2016ACA138), and the Fundamental Research Funds for the Central University, South-Central University for Nationalities (CZZ17006, CZQ17008). The authors thank Analytical \& Measuring Centre, South-Central University for Nationalities, for the NMR measurements.

\section{Compliance with Ethical Standards}

Conflict of interest The authors declare no competing financial interest.

Open Access This article is distributed under the terms of the Creative Commons Attribution 4.0 International License (http://creative commons.org/licenses/by/4.0/), which permits unrestricted use, distribution, and reproduction in any medium, provided you give appropriate credit to the original author(s) and the source, provide a link to the Creative Commons license, and indicate if changes were made.

\section{References}

1. U.R. Abdelmohsen, K. Bayer, U. Hentschel, Nat. Prod. Rep. 31, 381-399 (2014)

2. O. Genilloud, Nat. Prod. Rep. 34, 1203-1232 (2017)

3. P.B. Gupta, T.T. Onder, G. Jiang, K. Tao, C. Kuperwasser, R.A. Weinberg, E.S. Lander, Cell 138, 645-659 (2009)

4. Y. Miyazaki, M. Shibuya, H. Sugawara, O. Kawaguchi, C. Hirose, J. Nagatsu, S. Esumi, J. Antibiot. 27, 814-821 (1974)

5. L. Lazzarini, G. Cavaletti, F. Toppo, Marinelli. Antonie Van Leeuwenhoek 78, 399-405 (2000)

6. J. Clardy, M.A. Fischbach, C.T. Walsh, Nat. Biotechnol. 24, 1541-1550 (2006)

7. N.A. Krasil'nikov, L.V. Kalakutskii, N.F. Kirillova, Bull. Acad. Sci. USSR Ser. Biol. 1, 107-112 (1961)

8. M. Izumikawa, M. Takagi, K. Shin-Ya, J. Antibiot. 64, 689-691 (2011)

9. S. Qin, J.H. Jiang, H.P. Klenk, W.Y. Zhu, G.Z. Zhao, L.X. Zhao, S.K. Tang, L.H. Xu, W.J. Li, Int. J. Syst. Evol. Micr. 62, 84-89 (2012)

10. H. Fujimoto, Y. Okamoto, E. Sone, S. Maeda, K. Akiyama, M. Ishibashi, Chem. Pharm. Bull. 53, 923-929 (2005)

11. T. Mosmann, J. Immunol. Methods 65, 55-63 (1983)

12. L.J. Reed, H. Muench, Am. J. Hyg. 27, 493-497 (1938) 